\title{
Modern biotechnological approaches for improvement of nutritional value of grain sorghum
}

\author{
Elkonin L.A. ${ }^{1 *}$, Panin V.M. ${ }^{1}$, Gerashchenkov G.A. ${ }^{2}$ \\ ${ }^{1}$ Agricultural Research Institute of South-East Region, Saratov, Russia \\ ${ }^{2}$ Institute of Biochemistry and Genetics, UFRC RAS, Ufa, Russia \\ *e-mail:lelkonin@gmail.com
}

One of the promising areas of biotechnology is the improvement of nutritional value of grain. This task is particularly important for sorghum - a high-yielding drought-tolerant cereal, which is a source of feed and food for millions of people in many countries around the world. However, compared with other cereals, majority of sorghum cultivars have a lower nutritional value, which is based on the resistance of sorghum prolamines (mainly, gamma-kafirin) to proteolytic digestion. Recently, in several laboratories in different countries, transgenic sorghum lines with genetic constructs capable of RNAisilencing of kafirin genes were obtained. These lines were characterized by reduction of kafirin oligomers, an increase of in vitro digestibility of endosperm proteins, changes in the ultrastructure of endosperm protein bodies, which acquire an irregular shape with invaginations, and modification of endosperm texture, namely, reduction of the vitreous layer. Moreover, an important consequence of the silencing of the kafirin genes was the enhancement of synthesis of other proteins, including those that have a higher content of essential amino acids, lysine and threonine. Such a rebalancing of seed proteome is a common phenomenon for transgenic plants with genetic constructs for RNA-silencing of prolamine genes. In our experiments, in the offspring of plants with high protein digestibility, the introduced genetic construct functioned irregularly; in the course of plant ontogenesis, including the plants from the late generations $\left(\mathrm{T}_{4}, \mathrm{~T}_{5}\right)$, the elimination of the construct for RNA-silencing was observed. In this regard, the methods of genome editing have significant advantages, since they allow inducing genetic changes in the structure of kafirin genes. We started the work on creation of sorghum lines with a modified nucleotide sequence of the gamma-kafirin gene. These lines should have improved endosperm proteins digestibility and a higher level of essential amino acids. Acknowledgements: This work was partially supported by RFBR, grant No. 19-01600117. 